\title{
Senescent hepatocytes enhance natural killer cell activity via the CXCL-10/CXCR3 axis
}

\author{
JINFENG ZANG ${ }^{1}$, JUN YE ${ }^{2}$, CHI ZHANG ${ }^{1}$, MIN SHA $^{2}$ and JUNYE GAO ${ }^{1}$ \\ ${ }^{1}$ Department of Hepatobiliary Surgery; ${ }^{2}$ Central Laboratory, Taizhou People's Hospital, \\ The Fifth Affiliated Hospital of Nantong University Medical School, Taizhou, Jiangsu 225300, P.R. China
}

Received December 23, 2018; Accepted August 15, 2019

DOI: $10.3892 /$ etm.2019.8037

\begin{abstract}
Cellular senescence and natural killer (NK) cells play an important role in liver diseases. Chemokines, a component of the senescence-associated secretory phenotype, can recruit NK cells and are involved in the development of various liver diseases. The effect of the $\mathrm{C}-\mathrm{X}-\mathrm{C}$ motif chemokine ligand (CXCL)-9, -10, -11/C-X-C motif chemokine receptor (CXCR)3 axis in senescent hepatocytes remains unknown. The chemokines secreted by senescent hepatocytes, the contribution of the CXCL-9, -10, -11/CXCR3 axis to the migration of NK cells, and the effect of senescent hepatocytes on the function of NK cells were investigated in the present study. The results demonstrated significantly increased levels of C-C motif chemokine ligand 2 and CXCL-1, -2 and -10 in the supernatant of senescent AML12 cells. Despite increased mRNA expression of CXCL-9, -10, and -11 in these cells, western blotting revealed significantly enhanced expression of only CXCL-10. The expression of CXCR3 on the surface of NK cells stimulated by senescent AML12 cells was upregulated (fold change, $>3$ ). Following incubation with the supernatant of senescent hepatocytes, both CD107a and interferon $\gamma$ expression in NK cells increased by $>2.5$-fold. The cytotoxic effect of NK cells was notably higher stimulated by senescent AML12 cells. Chemotaxis and blocking assays demonstrated that the senescent hepatocytes enhanced the migration of NK cells via the CXCL-10/CXCR3 axis. The present study suggests that senescent hepatocytes secrete various chemokines, including CXCL-10, resulting in the upregulation and activation of CXCR3 in NK cells and the enhancement of NK cell migration via the CXCL-10/CXCR3 axis.
\end{abstract}

Correspondence to: Dr Jinfeng Zang, Department of Hepatobiliary Surgery, Taizhou People's Hospital, The Fifth Affiliated Hospital of Nantong University Medical School, 366 Taihu Road, Taizhou, Jiangsu 225300, P.R. China

E-mail: xkfy1979@126.com

Key words: hepatocyte, natural killer cell, senescence, chemokine

\section{Introduction}

Cellular senescence of hepatocytes, cholangiocytes, stellate cells and immune cells is extensively involved in chronic liver diseases (1). Senescent cells exert an influence on their neighboring cells and microenvironment through a number of types of components, such as cytokines, chemokines and growth factors; this is referred to as the senescence-associated secretory phenotype (SASP) (2). There is accumulating evidence that the culture medium of senescent cells is enriched with chemokines $(3,4)$. Chemokines and their receptors can modulate the migration of immune cells (3).

The liver is enriched with immune cells, such as Kupffer cells, dendritic cells, natural killer (NK) cells and NKT cells. NK cells represent a population of lymphocytes with potent cytolytic activity against infectious microbes or tumor cells. These cells are the main component of innate immunity and are able to interact with other immune cells such as macrophages, monocytes, neutrophils and dendritic cells $(5,6)$. The function of NK cells is normally regulated by a wide variety of surface receptors that respond to various signaling molecules. C-X-C motif chemokine receptor $(\mathrm{CXCR}) 3$ is an important receptor expressed on the surface of NK cells. Cells with a high expression level of CXCR3 display an activated phenotype, exerting strong cytotoxic effects. C-X-C motif chemokine ligand (CXCL)- $9,-10$ and -11 are selective ligands for CXCR3 and are usually expressed at low levels in homeostatic conditions. In response to interferon (IFN) $-\gamma$ stimulation, the expression of CXCL-9, -10 and -11 is enhanced in monocytes, endothelial cells, fibroblasts, cancer cells and other cell types (7-9). The CXCL-9, -10, -11/CXCR3 axis takes part mainly in immune cell migration, differentiation and activation, supporting the hypothesis that this chemotaxis is involved in liver injury, repair and tumorigenesis (10-12). However, the effect of the CXCL-9, -10, -11/CXCR3 axis in hepatocellular senescence remains unknown.

As demonstrated by a previous study, $\mathrm{H}_{2} \mathrm{O}_{2}$ successfully induces hepatocyte AML12 cells to develop a senescent phenotype (13). In the present study, the chemokines secreted by senescent hepatocytes and the contribution of these cells to the modulation of the function and migration of NK cells were investigated. The role of CXCL-10/CXCR3 chemotaxis, particularly in the recruitment of NK cells, was explored. Moreover, the effect of senescent hepatocytes on the function of NK cells was investigated. 


\section{Materials and methods}

Animals. Male and female C57BL/6 mice (age, 8-12 weeks; weight, 21.3-24.9 g) were purchased from The Shanghai Experimental Animal Center (Shanghai, China). The total number of mice used in the present study was 12 (6 of each sex). All mice were housed under standard laboratory conditions in a temperature-controlled environment with a 12-h light/darkness cycle and received water and standard diet ad libitum. Mice were euthanized by cervical dislocation. The study was approved by The Ethics Review Committee for Animal Experimentation at Taizhou People's Hospital (Taizhou, China) and performed according to the relevant national guidelines.

Cell lines. The transforming growth factor $\alpha$ transgenic mouse hepatocyte AML12 cell line (Type Culture Collection of the Chinese Academy of Sciences) was maintained in DMEM/F12 medium supplemented with $5 \mu \mathrm{g} / \mathrm{ml}$ insulin, $5 \mu \mathrm{g} / \mathrm{ml}$ transferrin, $5 \mathrm{ng} / \mathrm{ml}$ selenium, $40 \mathrm{ng} / \mathrm{ml}$ dexamethasone, $10 \%$ fetal bovine serum (FBS) and antibiotics $(100 \mathrm{U} / \mathrm{ml}$ penicillin and $100 \mu \mathrm{g} / \mathrm{ml}$ streptomycin). The AML12 cells were incubated with $10 \mu \mathrm{M} \mathrm{H}_{2} \mathrm{O}_{2}$ in culture medium for $72 \mathrm{~h}$ to induce senescence, as previously described (13). The supernatant of the cells was then collected and frozen at $-80^{\circ} \mathrm{C}$ until use according to a previous study (13). The mouse hepatoma cell Hepa-1c1c7 cell line (Type Culture Collection of the Chinese Academy of Sciences) was maintained in $\alpha$-MEM supplemented in $10 \%$ FBS, $100 \mathrm{U} / \mathrm{ml}$ penicillin and $100 \mu \mathrm{g} / \mathrm{ml}$ streptomycin, at $37^{\circ} \mathrm{C}$ in a humidified atmosphere with $5 \% \mathrm{CO}_{2}$.

NK cell isolation from mouse spleens. Spleens were removed aseptically from C57BL/6 mice. Single-cell suspensions of splenocytes were generated by passing the spleen through a $100 \mathrm{~mm}$ nylon mesh (Corning, Inc.) and washed with PBS supplemented with $3 \%$ fetal calf serum (Sigma-Aldrich; Merck KGaA) and $2 \mathrm{mM}$ EDTA. Following lysis of erythrocytes using Pharm Lyse ${ }^{\mathrm{TM}}$ lysing solution (BD Biosciences) for $3 \mathrm{~min}$ at room temperature, splenocytes were washed and passed through a $40 \mu \mathrm{m}$ nylon mesh (Corning, Inc.). Single-cell suspensions were prepared in RPMI-1640 medium (Sigma-Aldrich; Merck KGaA) containing 10\% FBS (Sigma-Aldrich; Merck KGaA). NK cells were isolated from splenocytes using the mouse NK Cell Isolation kit (Miltenyi Biotec $\mathrm{GmbH}$ ) according to the manufacturer's protocol. Briefly, NK cells were sorted by negative selection using LS Columns and the MidiMACS ${ }^{\mathrm{TM}}$ Separator (both Miltenyi Biotec $\mathrm{GmbH}$ ) from spleen cell suspensions. The purity of NK cells was determined by flow cytometry using the BD FACS Aria II (BD Biosciences) with anti-NK1.1 and -CD3 antibodies. NK cells were incubated with allophycocyanin (APC)-conjugated anti-NK1.1 antibodies (1:50; cat. no. 550627) and FITC-conjugated anti-CD3 antibodies (1:20; cat. no. 553062) (both BD Biosciences) for $30 \mathrm{~min}$ at $4^{\circ} \mathrm{C}$ in the dark. Data were analyzed using the FlowJo software (version 7.5.5; Tree Star, Inc.). Following the isolation, the purity of the NK $1.1^{+} \mathrm{CD}^{-}$cells was consistently $>95 \%$. The viability of NK cells was evaluated by trypan blue (0.4\%) staining for $10 \mathrm{~min}$ and counted using a hemocytometer by light microscopy. NK cell viability was $98 \%$.
Cytokine array. A total of 111 different cytokines in the supernatants of the cultures were measured using the Mouse XL Cytokine Array kit (R\&D Systems, Inc.), according to the manufacturer's protocol. The dot blot membranes were developed by enhanced chemiluminescence horseradish peroxidase (HRP) substrate (Millipore; Merck KGaA). Data were analyzed using the ImageJ software (version 1.48; National Institutes of Health) and normalized to reference spots.

Enzyme-linked immunosorbent assay (ELISA). Levels of CXCL-9, -10 and -11 in cell supernatants were assayed using mouse ELISA DuoSet kits (cat. nos. DY492, DY466 and DY572; R\&D Systems, Inc.). ELISAs were performed according to the manufacturer's protocols, and absorbance was determined at $450 \mathrm{~nm}$ using the CLARIOstar microplate reader (BMG Labtech $\mathrm{GmbH}$ ). The concentrations were calculated using a standard curve line of best fit on the MARS Data Analysis software (BMG Labtech $\mathrm{GmbH}$ ).

Western blotting. Whole-cell lysates were prepared by lysing cell pellets in RIPA lysis buffer containing protease inhibitor cocktail (Roche Diagnostics). Total protein concentration was measured using Bicinchoninic Acid Protein Assay Kit (Pierce; Thermo Fisher Scientific, Inc.). Equal amounts of protein $(20 \mu \mathrm{g})$ were subjected to $8 \%$ SDS-PAGE and transferred to polyvinylidene fluoride membranes (EMD Millipore; Merck KGaA), which were then blocked using TBS containing Tween-20 (0.1\%) and 5\% milk powder for $1 \mathrm{~h}$ at room temperature. The membranes were incubated with monoclonal anti-CXCL-9 (1:1,000; cat. no. 701117) and polyclonal anti-CXCL-10 (1:1,000; cat. no. PA5-79104) and anti-CXCL-11 (1:1,000; cat. no. PA5-47767) antibodies (all from Invitrogen; Thermo Fisher Scientific, Inc.) at $4^{\circ} \mathrm{C}$ overnight. Subsequently, the membranes were incubated with HRP-conjugated secondary antibody $(1: 1,000$; cat. no. G-21040; Invitrogen; Thermo Fisher Scientific, Inc.) for $2 \mathrm{~h}$ at room temperature. Bound secondary antibody was detected using enhanced chemiluminescence reagent (ECL; Millipore; Merck KGaA) and captured using an Image Quant LAS-4000 (Fujifilm Corporation). Protein quantification was normalized to $\beta$-actin (antibody dilution, 1:1,000; cat. no. MA5-15739; Invitrogen; Thermo Fisher Scientific, Inc.).

Reverse transcription-quantitative polymerase chain reaction $(R T-q P C R)$. Total RNA from harvested cells was extracted using the RNA isolation kit (BioTeke Corporation). Following assessment of quantity and quality by spectrophotometry, the RNA samples were reverse transcribed into cDNA using PrimeScript ${ }^{\mathrm{TM}}$ RT-PCR Kit (Takara Biotechnology Co., Ltd.) according to the manufacturer's instructions. The qPCR assays using SYBR ${ }^{\mathrm{TM}}$ Green PCR Master Mix (Thermo Fisher Scientific, Inc.) were performed in an ABI PRISM 7000 Sequence Detection system (Applied Biosystems; Thermo Fisher Scientific, Inc.) under the following thermocycling conditions: $50^{\circ} \mathrm{C}$ for $2 \mathrm{~min}, 95^{\circ} \mathrm{C}$ for $10 \mathrm{~min}$ and 40 cycles of $95^{\circ} \mathrm{C}$ for $15 \mathrm{sec}$ and $60^{\circ} \mathrm{C}$ for $1 \mathrm{~min}$. Data were normalized to the control GAPDH and analyzed by the $2^{-\Delta \Delta C q}$ method (14). Samples were assayed in triplicates in three independent experiments. The primers for the amplification of the indicated genes were as follows: CXCL-9 forward, 
5'-GAAGCAGCCAAGTCGGTTAGTG-3' and reverse, 5'-AATCATCAGCAGTGTGAGCAGTG-3'; CXCL-10 forward, 5'-TTAGTGGATGTTCTGACCCTGCTTC-3'; and reverse, 5'-TGGGAGGATGGCAGTGGAAGTC-3'; CXCL-11 forward, 5'-CCATCGGAGTTTACAAAGTGCT-3'; and reverse, 5'-TCTCCACCGTAACCACAGATAGC-3'; GAPDH forward, 5'-GCACCGTCAAGGCTGAGAAC-3' and reverse, 5'-TGGTGAAGACGCCAGTGGA-3'.

Functional analysis of $\mathrm{NK}$ cells by flow cytometry and cytotoxicity assay. NK cells $\left(1 \times 10^{6}\right)$ were treated with supernatant from senescent or normal AML12 cells for $72 \mathrm{~h}$. Following incubation with phycoerythrin (PE)-conjugated anti-CXCR3 (1:20; cat. no. 562152), FITC-conjugated anti-Ly49D (1:10; cat. no. 555313), PE-conjugated anti-CD107a (1:10; cat. no. 558661) and APC-conjugated anti-IFN- $\gamma$ antibodies (1:20; cat. no. 562018) (all BD Biosciences) for $30 \mathrm{~min}$ at $4^{\circ} \mathrm{C}$ in the dark, cells were washed with and resuspended in ice-cold PBS. Fc receptors were blocked with $30 \mathrm{mg} / \mathrm{ml}$ mouse IgG (cat. no. 31205; Invitrogen; Thermo Fisher Scientific, Inc.) and $10 \mathrm{mg} / \mathrm{ml}$ anti-CD16/32 (cat. no. 101302; BioLegend, Inc.) for $20 \mathrm{~min}$ at $4^{\circ} \mathrm{C}$. For the analysis of IFN- $\gamma$, permeabilization with 1X BD FACS Permeabilizing Solution 2 (BD Biosciences) for $10 \mathrm{~min}$ at room temperature was conducted prior to intracellular staining. The percentage of positive cells was determined using a FACS LSR flow cytometer (BD Biosciences).

The cytotoxicity of NK cells towards Hepa-1c1c7 target cells was assessed using the Cytotox 96 non-radioactive assay (Promega Corporation). Target cells were seeded on a 96-well plate at a density of 5,000 cells/well. NK cells were added at effector-to-target (E:T) ratios of 5:1, 10:1 and $20: 1$ and incubated at $37^{\circ} \mathrm{C}$ for $4 \mathrm{~h}$. The supernatant was harvested for analysis of cytotoxicity according to the release of lactate dehydrogenase. The killing effect of NK cells against target cells was assessed using the following equation: Cytotoxicity $=($ Experimental-Effector spontaneous-Target spontaneous)/(Target maximum-Target spontaneous) $\mathrm{x} 100 \%$.

Chemotaxis and blocking assays. Cell-free culture supernatants $(600 \mu \mathrm{l})$ from normal and senescent AML12 cells were added to the lower chambers of Transwell inserts (Corning Inc.). A solution of DMEM/F12/10\% FBS $(600 \mu 1)$ was used as the negative control. Alternatively, DMEM/F12/10\% FBS containing recombinant CXCL-10 (10 ng/ml; R\&D Systems, Inc.) was chosen as the positive control. NK cells $\left(1 \times 10^{5}\right)$ were plated in the upper chambers of Transwell inserts. The upper and lower chambers were separated by a polyester membrane with $5-\mu \mathrm{m}$ pores. The chambers were incubated for $8 \mathrm{~h}$ at $37^{\circ} \mathrm{C}$ in a humidified atmosphere containing $5 \% \mathrm{CO}_{2}$, and migrated NK cells in the lower chamber were counted by flow cytometry using the BD FACSAria ${ }^{\mathrm{TM}}$ II flow cytometer. Briefly, the content of the bottom chamber was collected and migrated $\mathrm{NK}$ cells were identified on gate $\mathrm{NK} 1.1^{+} \mathrm{CD}^{-}$. The absolute numbers of NK cells were estimated using BD TruCount ${ }^{\mathrm{TM}}$ tubes (BD Biosciences) based on the formula: [cell $]=\left(\mathrm{n}^{\circ}\right.$ events counted per test $\mathrm{x} \mathrm{n}^{\circ}$ beads per test $) /\left(\mathrm{n}^{\circ}\right.$ events in bead region $\mathrm{x}$ test volume). A total of 10,000 beads were routinely collected to analyze the populations.
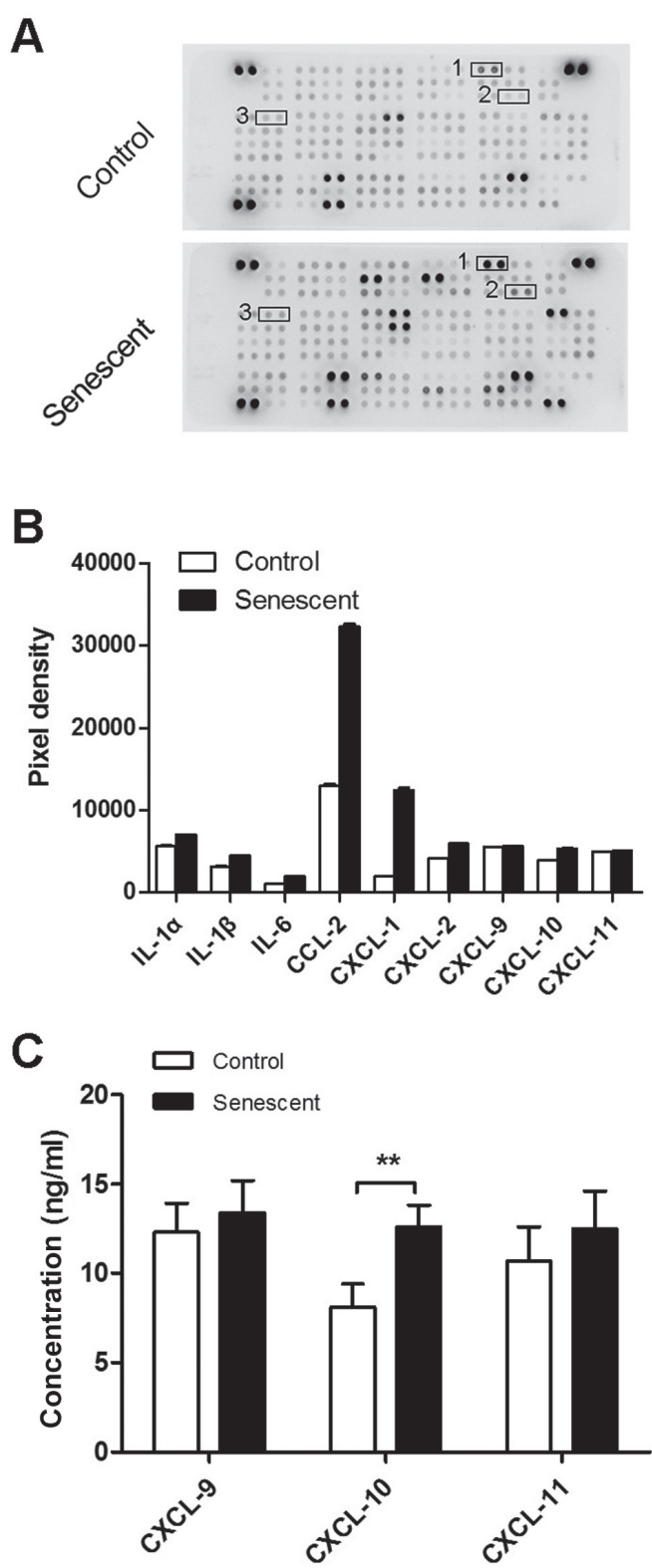

Figure 1. Cytokines in cell-free culture supernatants of senescent hepatocytes. (A) Representative images of cytokine arrays. 1, CCL-2; 2, CXCL-1; 3, CXCL-10. (B) Bar graph representing the densitometry of the spotted antibody array results. The secretion of IL-1 $\alpha$, IL-1 $\beta$, IL-6, CCL-2, CXCL-1 and CXCL-2 was differentially increased. (C) ELISA analysis of CXCL-9, -10 and -11 . The levels of only CXCL-10 exhibited significant augmentation. $\mathrm{n}=5$. Data were analyzed by Student's t-test. ${ }^{* *} \mathrm{P}<0.01$. IL, interleukin; $\mathrm{CCL}$, $\mathrm{C}-\mathrm{C}$ motif chemokine ligand; CXCL, C-X-C motif chemokine ligand.

Blocking of CXCR 3 on NK cells was performed by incubating $1 \times 10^{5} \mathrm{NK}$ cells with anti-CXCR3 monoclonal antibody $(20 \mu \mathrm{g} / \mathrm{ml})$ for $30 \mathrm{~min}$ at $37^{\circ} \mathrm{C}$. In another blocking experiment, the cell supernatants were neutralized with anti-CXCL-10 monoclonal antibody $(20 \mu \mathrm{g} / \mathrm{ml}$; cat. no. AF-466-NA; R\&D Systems, Inc.) for $30 \mathrm{~min}$ at $37^{\circ} \mathrm{C}$. The migration of NK cells to the lower chambers was evaluated as above. Data are presented as percentage of migration based on total cell input. Experiments were performed in triplicate.

Statistical analysis. SPSS (version 19.0; IBM Corp) was used to perform statistical analyses. For each independent 

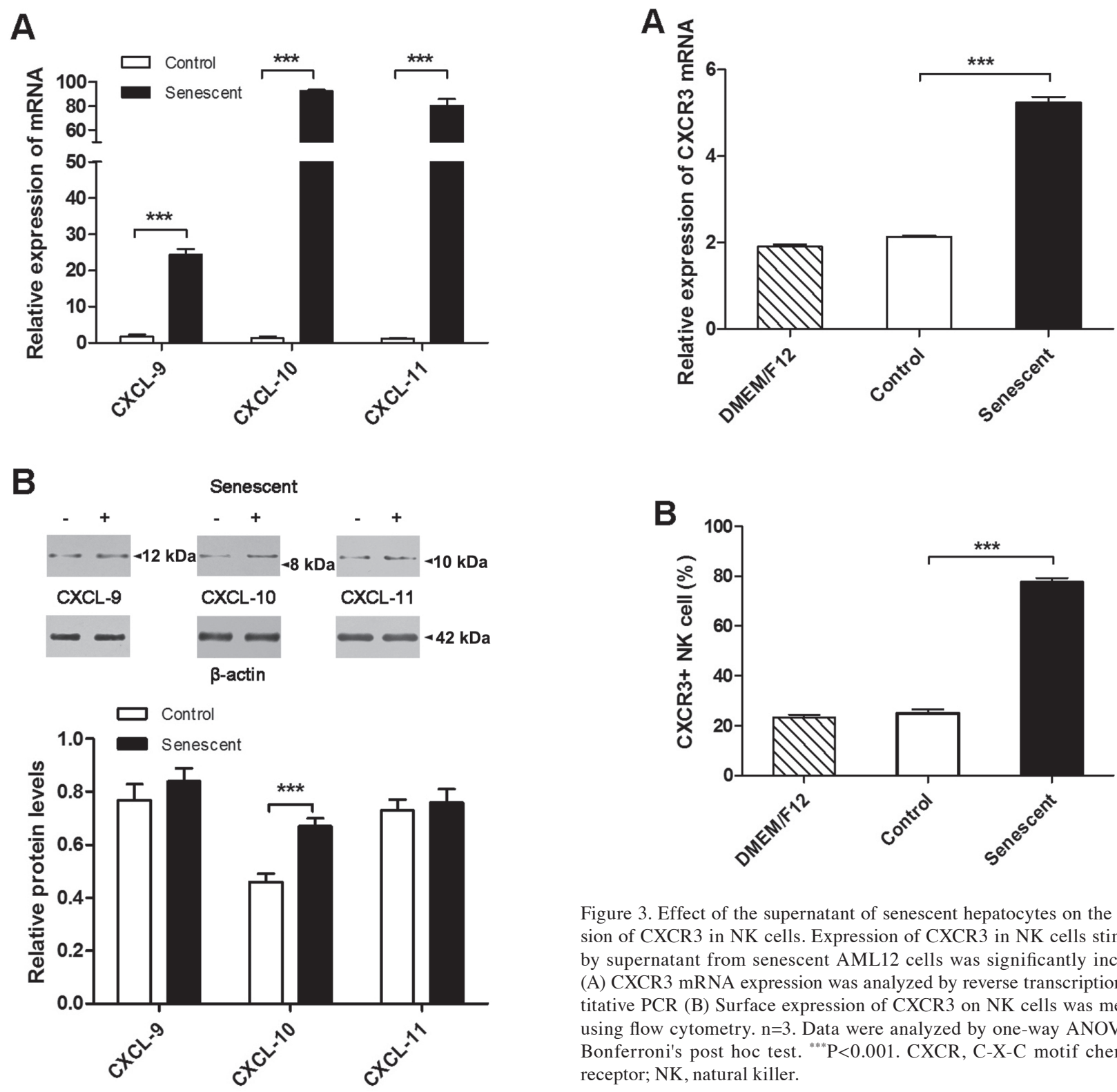

Figure 2. Expression of CXCL-9, -10 and -11 in senescent hepatocytes. (A) Expression of mRNA in senescent AML12 cells was measured by reverse transcription-quantitative PCR in comparison with controls. (B) Relative protein levels in senescent AML12 cells were determined by western blotting. $\mathrm{n}=3$. Data were analyzed by Student's t-test; ${ }^{* * *} \mathrm{P}<0.001$. CXCL, C-X-C motif chemokine ligand.

experiment, a minimum number of three experiments were performed to ensure reproducibility and adequate statistical power. Differences between two groups were compared by Student's t-test. Multiple group comparisons were made by one-way ANOVA followed by Bonferroni or Tukey's test. $\mathrm{P}<0.05$ was considered to indicate a statistically significant difference.

\section{Results}

Senescence phenotype in senescent hepatocytes. A previous study revealed that AML12 cells treated with $\mathrm{H}_{2} \mathrm{O}_{2}$ demonstrated typical morphological changes and a characteristic secretory phenotype (13). Senescent cells are able to

crosstalk with other cells through several secretory factors; this phenomenon is known as SASP. In order to gain a better understanding of the phenotype of senescent AML12 cells, the presence of 111 different cytokines were examined in the cell-free culture supernatants of $\mathrm{H}_{2} \mathrm{O}_{2}$-treated AML12 cells, using a cytokine array (Fig. 1A). The canonical SASP factors interleukin (IL)- $1 \alpha$, IL-1 $\beta$, IL- 6, C-C motif chemokine ligand (CCL)-2/monocyte chemoattractant protein-1, CXCL-1/growth related oncogene (GRO)- $\alpha$ and CXCL-2/GRO- $\beta$ were markedly increased in senescent compared with normal AML12 cells (Fig. 1B). Among these tested factors, CXCL-9, -10 and -11 , which are known to be CXCR3 ligands, were also detected by ELISA. In the culture supernatants from senescent AML12 cells, only the concentration of CXCL-10 exhibited significant augmentation compared with the levels from the control group (Fig. 1C).

CXCL-9, -10 and -11 expression levels in senescent hepatocytes. In order to confirm the results from the cytokine arrays of supernatants, the expression of CXCL-9, 
A

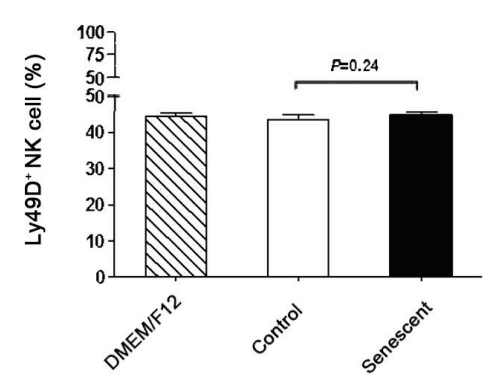

\section{Control}

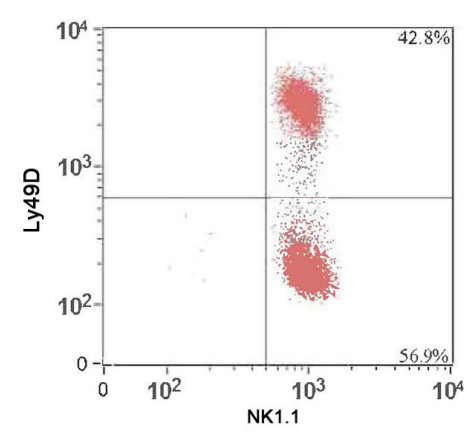

\section{Senescent}

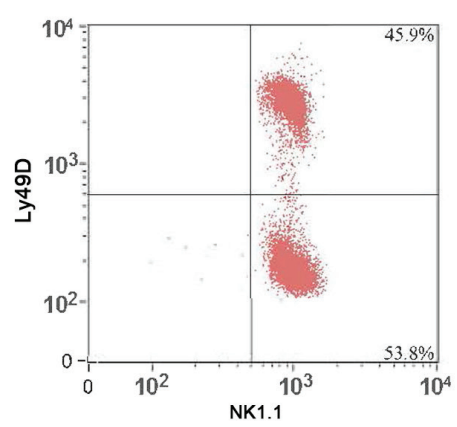

B
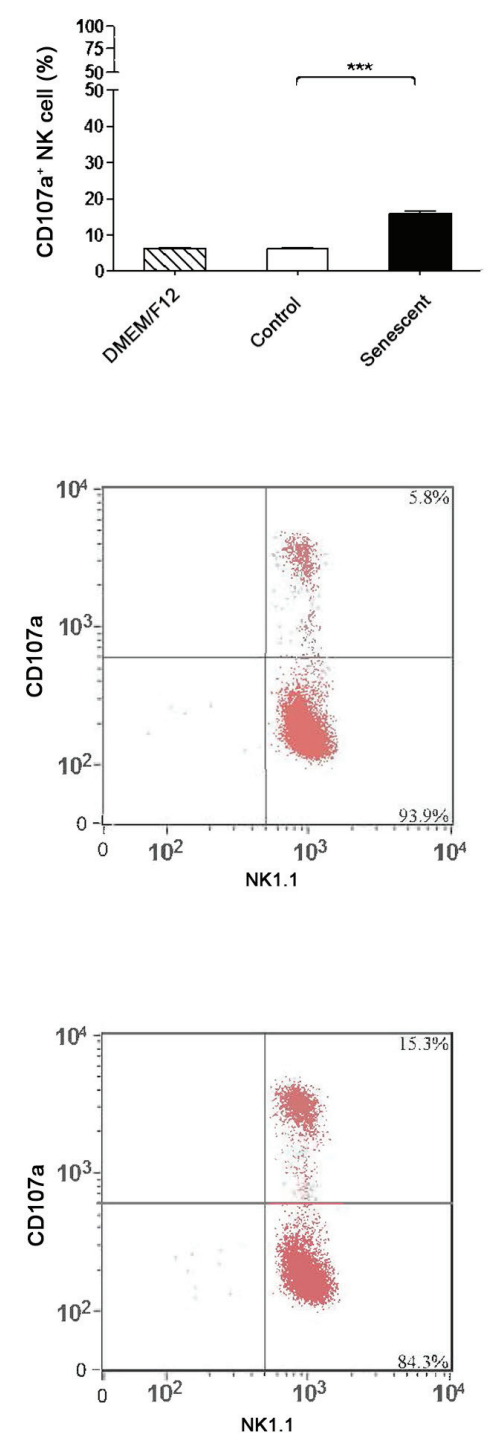

C
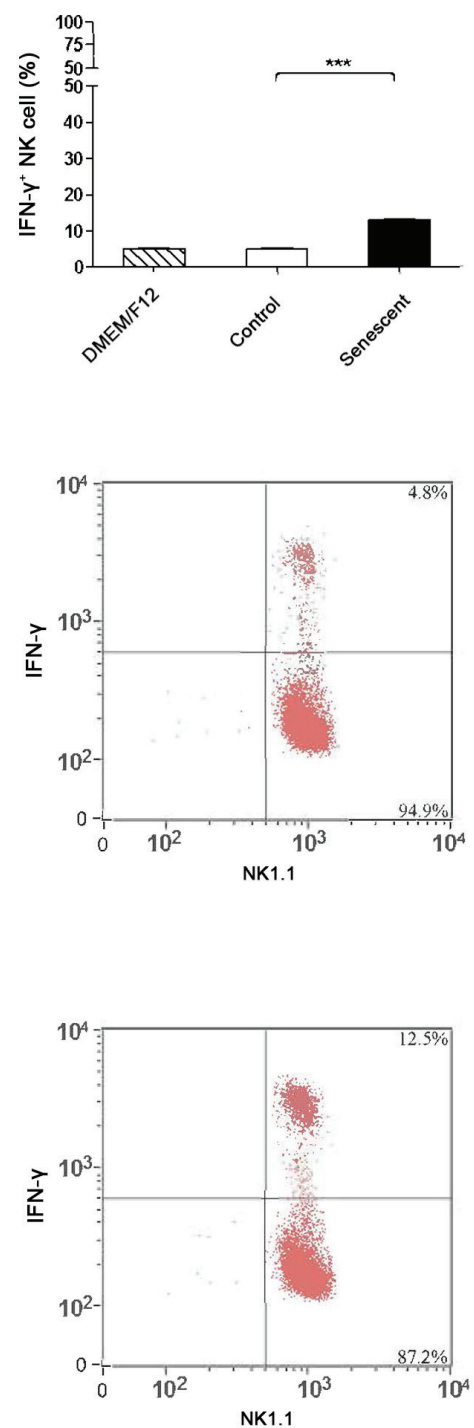

Figure 4. Effects of the supernatant of senescent hepatocytes on the expression of Ly49D, CD107a and IFN- $\gamma$ in NK cells. NK cells were treated with supernatant from senescent or normal AML12 cells for $72 \mathrm{~h}$. Flow cytometry analysis of the cell surface expression of (A) Ly49D and (B) CD107a. (C) Flow cytometry analysis of intracellular expression of IFN- $\gamma . n=3$. Data were analyzed by one-way ANOVA with Bonferroni's post hoc test. ${ }^{* * *} \mathrm{P}<0.001$. NK, natural killer; IFN, interferon.

-10 and -11 in senescent AML12 cells was further assessed using RT-qPCR and western blotting compared with non-senescent control cells. The mRNA expression level of these factors was increased in the senescent AML12 cells (Fig. 2A). However, western blotting showed significantly enhanced expression of only CXCL-10 (Fig. 2B). Therefore, it can be postulated that CXCL-10, as a CXCR3 ligand, is involved in the chemotactic recruitment of immune cells.

Senescent hepatocytes stimulate the expression of CXCR3 in $N K$ cells. As NK cells express several chemokine receptors exerting an important defensive function, it was hypothesized that chemokine signaling was involved in the trafficking of NK cells in the senescent liver. RT-qPCR was performed to determine the expression of CXCR3 mRNA in NK cells treated with cell culture supernatant from senescent AML12 cells. The expression levels were significantly increased following treatment with the supernatants of senescent AML12 cells
(Fig. 3A). The expression of CXCR3 on the surface of NK cells stimulated by senescent hepatocyte supernatant, as determined by flow cytometry, was $77.8 \pm 1.48 \%$, compared with $24.9 \pm 1.60 \%$ on the resting NK cells (Fig. 3B).

Effects of senescent hepatocytes on NK cell activity and function. To investigate the effects of senescent AML12 cells on NK cell function, the surface expression of markers of NK cell activation Ly49D and CD107a, and the intracellular expression of IFN- $\gamma(15,16)$, were determined. NK cells stimulated with the supernatant of senescent AML12 cells showed significantly increased expression of CD107a and IFN $-\gamma$. Following incubation with the supernatant of senescent AML12 cells, CD107a expression on the surface of NK cells increased $>2.5$-fold, resulting in $15.9 \%$ of NK cells expressing $\mathrm{CD} 107 \mathrm{a}$. The intracellular expression of IFN- $\gamma$ was also upregulated 2.5-fold. However, there was no significant increase in the expression level of Ly49D (Fig. 4). 
In order to demonstrate the cytotoxic effect of $\mathrm{NK}$ cells stimulated by the supernatant of senescent AML12 cells in vitro, $\mathrm{NK}$ cells were co-incubated with Hepa-1c1c7 cells at E:T ratios of 5:1, 10:1 and 20:1. At each ratio, the treated NK cells exhibited significantly increased cytotoxic effects compared with the control group (Fig. 5).

Senescent hepatocytes enhance migration of $N K$ cells via the CXCL-10/CXCR3 axis. To test the chemotactic activity of NK cells affected by senescent hepatocytes, NK cells were plated in a chamber assay, and their migratory response to the culture supernatant of senescent AML12 cells was evaluated. The migrating NK cells were $1.73 \pm 0.14 \%$ to untreated DMEM/F12 medium, $1.65 \pm 0.18 \%$ to normal AML12 cells, and $19.6 \pm 0.32 \%$ to senescent AML12 cells (Fig. 6). The migration of NK cells was 12-fold higher towards senescent AML12 cells compared with that to normal hepatocytes. To determine the effect of the CXCL-10/CXCR3 axis on the migration of NK cells to senescent AML12 cells, anti-CXCR3 or anti-CXCL-10 antibodies were used to neutralize the effect of these proteins on chemotaxis. The addition of these antibodies significantly inhibited the migration of NK cells to the supernatant of senescent AML12 cells.

\section{Discussion}

In vitro and in vivo experiments in cell lines, animal models and humans have demonstrated that senescence of hepatocytes, cholangiocytes, stellate cells and immune cells is involved in a wide spectrum of chronic liver disorders (17-20). In chronic viral hepatitis B and C, alcohol-related liver disease and non-alcohol-related fatty liver disease, senescent phenotype of hepatocytes is clearly detectable within the liver parenchyma (21-24). Senescent hepatocytes have been shown to accumulate with ongoing liver insult. Given the anti-apoptotic nature of senescent cells, senescent hepatocytes are likely to persist for a long period. During advanced stages of liver disease, the liver undergoes an enormous burden of senescence, since as many as $80 \%$ of hepatocytes are in this state (25). As senescent cells can be eliminated by attracting both adaptive and innate immune cells, senescence is a dynamic process (26-27). The lack of immune-mediated clearance of senescent hepatocytes in chronic liver diseases is likely to contribute to the clustering of these cells. The recruitment of immune cells for the clearance of cell debris and senescent cells plays a crucial role in wound healing. Moreover, immune clearance of senescent cells can markedly decrease the incidence of hepatocellular carcinoma development (28). A previous study using a mouse model reported that monocytes/macrophages orchestrated by $\mathrm{CD} 4^{+} \mathrm{T}$ cells executed the clearance of senescent hepatocytes, which inhibited the development of liver tumor (28).

It is widely accepted that senescent cells have a considerable impact on their microenvironment through SASP factors. SASP acts as a messenger between senescent cells and neighboring cells, contributing to tissue repair, inflammation and tumorigenesis. The most prominent cytokines of the SASP are IL-1, IL-6 and IL-8. Expression of IL-6 and IL-8 can be enhanced by IL-1, indicating a hierarchy of SASP regulation. IL-1 can promote the development of a senescent phenotype in neighboring cells through paracrine activity (29). IL-6 and IL-8 act as an autocrine feedback loop and strengthen senescence

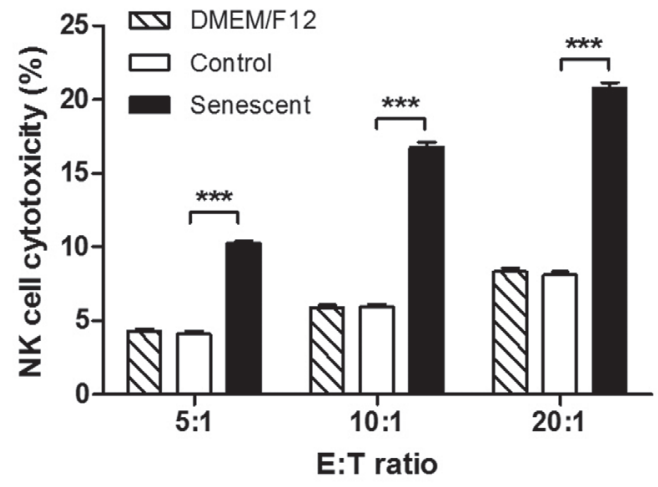

Figure 5. NK cell cytotoxicity for Hepa-1c1c7 cells. NK cells stimulated by supernatant from senescent AML12 cells were co-incubated with Hepa-1c1c7 cells at E:T ratios of 5:1, 10:1 and 20:1 for $4 \mathrm{~h}$. The cytotoxic activity of NK cells was examined using the CytoTox 96 Non-Radioactive Cytotoxicity Assay system. $n=5$. Data were analyzed by one-way ANOVA with Bonferroni's post hoc test. ${ }^{* * *} \mathrm{P}<0.001$. NK, natural killer; CXCR, C-X-C motif chemokine receptor; CXCL, C-X-C motif chemokine ligand; E, effector; T, target.

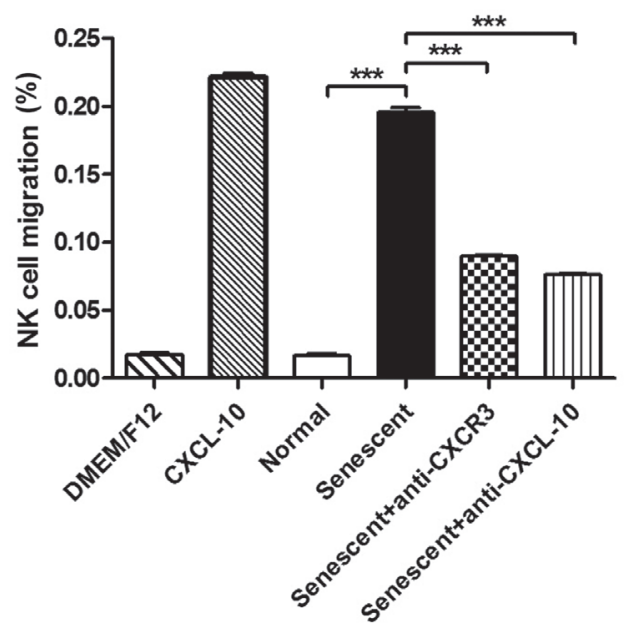

Figure 6. Migration of NK cells in response to supernatants from senescent AML12 cells. Anti-CXCR3 or anti-CXCL-10 antibodies were used for the blocking assay. The percentage of NK cell migration was calculated by dividing the number of cells that migrated to the lower well by the total number of cells. $n=3$. Data were analyzed by one-way ANOVA with Tukey's post hoc test. ${ }^{* * *} \mathrm{P}<0.001$. NK, natural killer; CXCR, C-X-C motif chemokine receptor; CXCL, C-X-C motif chemokine ligand.

by halting growth. The present study revealed that senescent hepatocytes exhibit SASP, expressing various chemokines, such as CCL-2, CXCL-1, CXCL-2 and CXCL-10. Similarly, senescent biliary epithelial cells induced by oxidative stress, DNA damage or serum deprivation upregulate the expression of chemokines, including CCL2 and C-X3-C motif chemokine ligand 1 (CX3CL1). It was demonstrated that senescent biliary epithelial cells in primary biliary cirrhosis recruited monocytes by secreting CCL-2 and CX3CL1, and possibly participated in the modulation of the inflammatory microenvironment (30). Additionally, the present study demonstrated that senescent hepatocytes induced significant chemotaxis of NK cells, by secreting CXCL-10. It is of particular interest that only the protein level of CXCL-10 was significantly upregulated, despite increased mRNA expression of CXCL-9, -10 and -11 . The reason for the difference between protein and mRNA 
level lies in the fact that, following synthesis, certain SASP factors still undergo post-translational modifications prior to their paracrine actions. For example, during oncogene-induced senescence, the inflammasome (a protein complex formed by caspase 1 and accessory proteins) serves an important role in the activation of the IL-1-signaling pathway, by processing and activating IL-1 $\beta$ (31).

The results of the present study suggest that senescent hepatocytes participate in the adjustment of the microenvironment, by recruiting NK cells and possibly other types of immune cells via chemokines. NK cells are an important component of the innate immune system that rapidly responds to intracellular pathogens and tumors, through IFN- $\gamma$ secretion and perforin-dependent target cell elimination $(32,33)$. The cytotoxicity of NK cells relies on the directed release of the contents of lytic granules, which are specific secretory lysosomes that contain perforin, granzymes and Fas ligands $(34,35)$. The first step in response to pathogens involves the recruitment of NK cells to the site of infection or tumor. The selective expression of different chemokine receptors on the surface of NK cells regulates the hepatic recruitment of NK cells. Recent data demonstrated that CXCR3-mediated recruitment of functionally distinct NK cell subpopulations may serve a role in the regulation of infection and autoimmune hepatitis (36-37). A CXCR3-knockout mouse model showed that NK cells were significantly decreased at the site of tumor infiltration, and CXCL10-controlled NK cell recruitment was correlated with tumor cell suppression as well as improved prognosis (38).

In the present study, NK cells stimulated by senescent hepatocytes resulted in a 3 -fold increase in the expression of CXCR3. In addition, NK cells stimulated by senescent hepatocytes displayed a significant increase in IFN- $\gamma$ production and higher expression of the NK cell receptor CD107a. CD107a is associated with lysosomal membranes and the degranulation of NK cells, and is as a marker of activation (39). Thus, senescent hepatocytes may result in the upregulation of CXCR3 in NK cells and induce NK cell activation.

CXCL-10 is among the chemokines that mediate the migration of leukocytes to the pathological sites in various liver diseases, primarily through CXCR3 (11). The findings of the present study demonstrated that senescent hepatocytes enhanced the migration of NK cells in vitro through the CXCL-10/CXCR3 axis. Similarly, recent studies have shown that CXCL-10 is implicated in the pathogenesis of liver inflammation and tumor. For instance, CXCL-10 neutralization in mice fed with a methionine- and choline-deficient diet decreased lipid accumulation and inflammatory cell infiltration, which eventually prevented non-alcoholic steatohepatitis (NASH) (11). It was demonstrated in human NASH that circulating and hepatic CXCL-10 levels were significantly higher and were regarded as a potential noninvasive biomarker. A study on concanavalin A-induced hepatitis revealed increased release of CXCL-9, -10 and -11 in hepatic cell lines, following IL-27 stimulation, whereas CXCL-10 and CXCL-11 proteins were significantly augmented in the sera of mice (40).

In conclusion, the findings of the present study provide important insights into the CXCL-10/CXCR3 axis in senescent hepatocytes and NK cells. Senescent hepatocytes secrete various chemokines, including CXCL-10. NK cells, in response to senescent hepatocytes, induce the upregulation and activation of CXCR3. Furthermore, senescent hepatocytes enhance the migration of NK cells via the CXCL-10/CXCR3 axis. Therefore, targeting the CXCL-10/CXCR3 pathway may regulate NK cell mobilization towards senescent hepatocytes, and lead to a novel strategy for intervening senescence-associated pathologies in liver diseases.

\section{Acknowledgements}

Not applicable.

\section{Funding}

The present study was supported by The Science and Technology Bureau of Taizhou (grant no. TS201626) and The Taizhou People's Hospital (grant no. ZL201628).

\section{Availability of data and materials}

The datasets used and/or analyzed during the current study are available from the corresponding author on reasonable request.

\section{Authors' contributions}

JZ, JG and JY designed all the experiments. JZ, MS, CZ and JY performed the experiments. JZ and JG wrote the manuscript. All authors read and approved the final manuscript.

\section{Ethics approval and consent to participate}

The present study was approved by The Ethics Review Committee for Animal Experimentation at Taizhou People's Hospital, Taizhou, China.

\section{Patient consent for publication}

Not applicable.

\section{Competing interests}

The authors declare that they have no competing interests.

\section{References}

1. Aravinthan AD and Alexander GJM: Senescence in chronic liver disease: Is the future in aging? J Hepatol 65: 825-834, 2016.

2. Byun HO, Lee YK, Kim JM and Yoon G: From cell senescence to age-related diseases: Differential mechanisms of action of senescence-associated secretory phenotypes. BMB Rep 48: 549-558, 2015.

3. Irvine KM, Skoien R, Bokil NJ, Melino M, Thomas GP, Loo D, Gabrielli B, Hill MM, Sweet MJ, Clouston AD and Powell EE: Senescent human hepatocytes express a unique secretory phenotype and promote macrophage migration. World J Gastroenterol 20: 17851-17862, 2014.

4. Maciel-Barón LA, Morales-Rosales SL, Aquino-Cruz AA, Triana-Martínez F, Galván-Arzate S, Luna-López A, González-Puertos VY, López-Díazguerrero NE, Torres C and Königsberg M: Senescence associated secretory phenotype profile from primary lung mice fibroblasts depends on the senescence induction stimuli. Age (Dordr) 38: 26, 2016.

5. Bellora F, Castriconi R, Dondero A, Reggiardo G, Moretta L, Mantovani A, Moretta A, and Bottino C: The interaction of human natural killer cells with either unpolarized or polarized macrophages results in different functional outcomes. Proc Natl Acad Sci USA 107: 21659-21664, 2010. 
6. Allen F, Bobanga ID, Rauhe P, Barkauskas D, Teich N, Tong C Myers $\mathrm{J}$ and Huang AY: CCL3 augments tumor rejection and enhances $\mathrm{CD}^{+} \mathrm{T}$ cell infiltration through $\mathrm{NK}$ and $\mathrm{CD} 103^{+}$dendritic cell recruitment via IFN $\gamma$. Oncoimmunology 7: e1393598, 2017.

7. Bernardini G, Antonangeli F, Bonanni V and Santoni A: Dysregulation of chemokine/chemokine receptor axes and NK cell tissue localization during diseases. Front Immunol 7: 402, 2016.

8. Marquardt N, Wilk E, Pokoyski C, Schmidt RE and Jacobs R: Murine CXCR3+CD27bright NK cells resemble the human CD56bright NK-cell population. Eur J Immunol 40: 1428-1439, 2010.

9. Wang Z, Wu T, Ma M, Zhang Z, Fu Y, Liu J, Xu J, Ding H, Han X, Chu Z, et al: Elevated interferon- $\gamma$-induced protein 10 and its receptor CXCR3 impair NK cell function during HIV infection. J Leukoc Biol 102: 163-170, 2017.

10. Hintermann E, Bayer M, Pfeilschifter JM, Luster AD and Christen U: CXCL10 promotes liver fibrosis by prevention of NK cell mediated hepatic stellate cell inactivation. J Autoimmun 35 424-435, 2010

11. Zhang X, Shen J, Man K, Chu ES, Yau TO, Sung JC, Go MY, Deng J, Lu L, Wong VW, et al: CXCL10 plays a key role as an inflammatory mediator and a non-invasive biomarker of non-alcoholic steatohepatitis. J Hepatol 61: 1365-1375, 2014.

12. Ling CC, Ng KT, Shao Y, Geng W, Xiao JW, Liu H, Li CX, Liu XB, Ma YY, Yeung WH, et al: Post-transplant endothelial progenitor cell mobilization via CXCL10/CXCR3 signaling promotes liver tumor growth. J Hepatol 60: 103-109, 2014.

13. Zang J, Sha M, Zhang C, Ye J, Zhang K and Gao J: Senescent hepatocyte secretion of matrix metalloproteinases is regulated by nuclear factor- $\kappa$ B signaling. Life Sci 191: 205-210, 2017.

14. Livak KJ and Schmittgen TD: Analysis of relative gene expression data using real-time quantitative PCR and the 2(-Delta Delta C(T)) method. Methods 25: 402-408, 2001.

15. Bryceson YT, Chiang SC, Darmanin S, Fauriat C, Schlums H, Theorell J and Wood SM: Molecular mechanisms of natural killer cell activation. J Innate Immun 3: 216-226, 2011.

16. Rahim MM, Tu MM, Mahmoud AB, Wight A, Abou-Samra E Lima PD and Makrigiannis AP: Ly49 receptors: Innate and adaptive immune paradigms. Front Immunol 5: 145, 2014

17. Sasaki M, Ikeda H, Yamaguchi J, Miyakoshi M, Sato Y and Nakanuma Y: Bile ductular cells undergoing cellular senescence increase in chronic liver diseases along with fibrous progression. Am J Clin Pathol 133: 212-223, 2010.

18. Aravinthan AD and Alexander GJ: Hepatocyte senescence explains conjugated bilirubinaemia in chronic liver failure. J Hepatol 63: 532-533, 2015.

19. Jin H, Jia Y, Yao Z, Huang J, Hao M, Yao S, Lian N, Zhang F, Zhang C, Chen X, et al: Hepatic stellate cell interferes with NK cell regulation of fibrogenesis via curcumin induced senescence of hepatic stellate cell. Cell Signal 33: 79-85, 2017.

20. Barathan M, Mohamed R, Saeidi A, Vadivelu J, Chang LY, Gopal K, Ram MR, Ansari AW, Kamarulzaman A, Velu V, et al: Increased frequency of late-senescent T cells lacking CD127 in chronic hepatitis C disease. Eur J Clin Invest 45: 466-474, 2015.

21. Sekoguchi S, Nakajima T, Moriguchi M, Jo M, Nishikawa T, Katagishi T, Kimura H, Minami M, Itoh Y, Kagawa K, et al: Role of cell-cycle turnover and oxidative stress in telomere shortening and cellular senescence in patients with chronic hepatitis $\mathrm{C}$. J Gastroenterol Hepatol 22: 182-190, 2007.

22. Aravinthan A, Pietrosi G, Hoare M, Jupp J, Marshall A, Verrill C, Davies S, Bateman A, Sheron N, Allison M and Alexander GJ: Hepatocyte expression of the senescence marker p21 is linked to fibrosis and an adverse liver-related outcome in alcohol-related liver disease. PLoS One 8: e72904, 2013.

23. Tachtatzis PM, Marshall A, Arvinthan A, Verma S, Penrhyn-Lowe S, Mela M, Scarpini C, Davies SE, Coleman N and Alexander GJ: Chronic hepatitis B virus infection: The relation between hepatitis B antigen expression, telomere length, senescence, inflammation and fibrosis. PLoS One 10: e0127511, 2015.

24. Aravinthan A, Mells G, Allison M, Leathart J, Kotronen A, Yki-Jarvinen H, Daly AK, Day CP, Anstee QM and Alexander G: Gene polymorphisms of cellular senescence marker p21 and disease progression in non-alcohol-related fatty liver disease. Cell Cycle 13: 1489-1494, 2014.
25. Aravinthan A, Scarpini C, Tachtatzis $P$, Verma $S$, Penrhyn-Lowe S, Harvey R, Davies SE, Allison M, Coleman N and Alexander G: Hepatocyte senescence predicts progression in non-alcohol-related fatty liver disease. J Hepatol 58: 549-556, 2013.

26. Yu Q, Katlinskaya YV, Carbone CJ, Zhao B, Katlinski KV, Zheng $\mathrm{H}$, Guha $\mathrm{M}$, Li $\mathrm{N}$, Chen $\mathrm{Q}$, Yang $\mathrm{T}$, et al: DNA-damage-induced type I interferon promotes senescence and inhibits stem cell function. Cell Rep 11: 785-797, 2015.

27. Fülöp T, Dupuis G, Witkowski JM and Larbi A: The role of immunosenescence in the development of age-related diseases. Rev Invest Clin 68: 84-91, 2016.

28. Kang TW, Yevsa T, Woller N, Hoenicke L, Wuestefeld T, Dauch D, Hohmeyer A, Gereke M, Rudalska R, Potapova A, et al: Senescence surveillance of pre-malignant hepatocytes limits liver cancer development. Nature 479: 547-551, 2011.

29. Hubackova S, Krejcikova K, Bartek J and Hodny Z: IL1- and TGF $\beta$-Nox 4 signaling, oxidative stress and DNA damage response are shared features of replicative, oncogene-induced, and drug-induced paracrine 'bystander senescence'. Aging (Albany NY) 4: 932-951, 2012.

30. Sasaki M, Miyakoshi M, Sato Y and Nakanuma Y: Modulation of the microenvironment by senescent biliary epithelial cells may be involved in the pathogenesis of primary biliary cirrhosis J Hepatol 53: 318-325, 2010.

31. Acosta JC, Banito A, Wuestefeld T, Georgilis A, Janich P, Morton JP, Athineos D, Kang TW, Lasitschka F, Andrulis M, et al: A complex secretory program orchestrated by the inflammasome controls paracrine senescence. Nat Cell Biol 15: 978-990, 2013.

32. Vesely MD, Kershaw MH, Schreiber RD and Smyth MJ: Natural innate and adaptive immunity to cancer. Annu Rev Immunol 29: 235-271, 2011.

33. Bernardini G, Gismondi A and Santoni A: Chemokines and NK cells: Regulators of development, trafficking and functions. Immunol Lett 145: 39-46, 2012.

34. Shevtsov M and Multhoff G: Immunological and translational aspects of NK cell-based antitumor immunotherapies. Front Immunol 7: 492, 2016

35. Zhu Y, Huang B and Shi J: Fas ligand and lytic granule differentially control cytotoxic dynamics of natural killer cell against cancer target. Oncotarget 7: 47163-47172, 2016.

36. Eisenhardt M, Glässner A, Krämer B, Körner C, Sibbing B, Kokordelis P, Nischalke HD, Sauerbruch T, Spengler U and Nattermann J: The CXCR3(+)CD56Bright phenotype characterizes a distinct NK cell subset with anti-fibrotic potential that shows dys-regulated activity in hepatitis C. PLoS One 7: e38846, 2012.

37. Xiao F, Ai G, Yan W, Wan X, Luo X, and Ning Q: Intrahepatic recruitment of cytotoxic NK cells contributes to autoimmune hepatitis progression. Cell Immunol 327: 13-20, 2018.

38. Wendel M, Galani IE, Suri-Payer E and Cerwenka A: Natural killer cell accumulation in tumors is dependent on IFN-gamma and CXCR3 ligands. Cancer Res 68: 8437-8445, 2008.

39. Gómez-Lomelí P, Bravo-Cuellar A, Hernández-Flores G, Jave-Suárez LF, Aguilar-Lemarroy A, Lerma-Díaz JM, Domínguez-Rodríguez JR, Sánchez-Reyes $K$ and Ortiz-Lazareno PC: Increase of IFN- $\gamma$ and TNF- $\alpha$ production in CD107a+NK-92 cells co-cultured with cervical cancer cell lines pre-treated with the HO-1 inhibitor. Cancer Cell Int 14: 100, 2014.

40. Basset L, Chevalier S, Danger Y, Arshad MI, Piquet-Pellorce C, Gascan $\mathrm{H}$ and Samson M: Interleukin-27 and IFN $\gamma$ regulate the expression of CXCL9, CXCL10, and CXCL11 in hepatitis. J Mol Med (Berl) 93: 1355-1367, 2015.

This work is licensed under a Creative Commons Attribution-NonCommercial-NoDerivatives 4.0 International (CC BY-NC-ND 4.0) License. 